\title{
Rapid Identification of Candida albicans \\ Septicemia in Man by Gas-Liquid Chromatography
}

\author{
Geraldine G. Mitler, Michael W. Witwer, Abrafam I. Braude, and \\ Charles E. Davis \\ From the Departments of Medicine and Pathology, University of California at \\ San Diego, San Diego, California 92103
}

\begin{abstract}
A B S T R A C T Gas-liquid chromatography (GLC) was used to study normal serum and serum from patients with septicemia caused by a variety of bacteria and by Candida albicans. The gas chromatograms of seven sera from six patients with septicemia due to $C$. albicans were found to be significantly and reproducibly different from those of normal sera. Chromatograms of serum from 19 bacteremic patients were indistinguishable from normals. The major peaks present in chromatograms of normal sera were identified by GLC and mass spectroscopy as the methyl esters of palmitic, oleic, linoleic, and stearic acids. In addition to these peaks, serum from patients with candidemia contained abnormal peaks that were also present in cultures of $C$. albicans grown in normal serum and in washed $C$. albicans harvested from cultures in yeast nitrogen base broth. Chromatograms from 11 cases of mucosal candidiasis differed little from normal and were easily distinguished from those of fungemia patients. Chromatograms of serum from two of four patients with deep-invasive candidiasis were indistinguishable from those of fungemia and reverted to normal after infections were eradicated.
\end{abstract}

\section{INTRODUCTION}

If the mortality rate from serious infections is to be lowered by prompt treatment, a rapid method of identifying the pathogenic organism is needed. Gas-liquid

Received for publication 7 November 1973 and in revised form 4 August 1974.

The Journal of Clinical Investigation Volume 54 November 1974-1235-1240 chromatography (GLC) ${ }^{1}$ has been used for the analysis of bacterial cell components $(1,2)$ and fermentation products (3). More recently, Mitruka and coworkers have identified the cause of bacteremia in experimental animals by a profile of the gas chromatogram of serum $(4,5)$. We have been unable to confirm these results in the present study but report promising new findings in the rapid diagnosis of Candida septicemia.

\section{METHODS}

Normal serum, obtained from 16 healthy medical students and laboratory personnel, was allowed to clot in a sterile glass tube for $1 \mathrm{~h}$, separated by centrifugation, and frozen at $-20^{\circ} \mathrm{C}$ until immediately before use. Blood was obtained, during the course of drawing blood for culture, from hospitalized patients suspected of septicemia. 26 sera corresponding to seven episodes of Candida albicans sepsis and 19 cases of bacteremia, and 5 sera from proven cases of leprosy were selected for study from the frozen samples. Blood was also drawn from 11 patients with proven superficial candidiasis and sterile blood cultures and from 4 patients with deep-invasive candidiasis. The group of deepinvasive infections was composed of a sternal wound infection with $C$. albicans after cardiac valvular surgery, scrotal candidiasis secondary to a Scott periurethral prosthesis, pyelitis and papillary necrosis due to $C$. albicans cultured from the papilla at pyelostomy, and $C$. albicans colitis. Serum was obtained, processed, and stored exactly as for the normals.

Cultures of $C$. albicans in serum were made from a laboratory strain originally isolated from human blood. $10-\mathrm{ml}$

${ }^{1}$ Abbreviations used in this paper: GLC, gas-liquid chromatography; TMS, trimethylsilyl; YNB, yeast nitrogen base broth.

\footnotetext{
The Journal of Clinical Investigation Volume 54 Nowember $1974 \cdot 1235-1240$
} 
aliquots of heat-inactivated normal human serum were inoculated with $0.1 \mathrm{ml}$ of a $72-\mathrm{h}$ culture of the yeast in a yeast nitrogen base broth (YNB). The culture and control $(10 \mathrm{ml}$ heat-inactivated normal serum plus $0.1 \mathrm{ml}$ sterile broth) were incubated at $37^{\circ} \mathrm{C}$ for $72 \mathrm{~h}$ and centrifuged at $3,000 \mathrm{rpm}$ for $15 \mathrm{~min}$, and the supernate was passed through a $0.45-\mu \mathrm{m}$ Millipore filter. The cell-free serum and control were then prepared for chromatography in the same way as the other specimens.

Cultures of $C$. albicans in yeast nitrogen base broth were also studied. Cells from a $50-\mathrm{ml}$ overnight culture were collected by centrifugation at $10,000 \mathrm{~g}$, washed three times in normal saline, dried overnight in a vacuum oven at $40^{\circ} \mathrm{C}$, suspended in $1 \mathrm{ml}$ distilled water, and prepared for chromatography in the same way as other specimens. 1-ml samples of the whole culture and the first wash were also chromatographed.

$1 \mathrm{ml}$ of serum was mixed with an equal volume of $8 \mathrm{~N}$ methanolic $\mathrm{HCl}$ in a screwcapped vial with a Teflon liner to prevent extraction of the cap. The sample was heated for $1 \mathrm{~h}$ at $80^{\circ} \mathrm{C}$ in a water bath to prepare O-methyl esters. The resulting hydrolysate was neutralized to $\mathrm{pH} 7.0$ with concentrated $\mathrm{NH}_{4} \mathrm{OH}$ and taken to dryness at $40^{\circ} \mathrm{C}$ for approximately $45 \mathrm{~min}$ in a flash evaporator (Buchler Instruments Div., Nuclear-Chicago Corp., Fort Lee, N. J.). After the dried material was taken up in $1.5 \mathrm{ml}$ pyridine and transferred to a glass tube, $0.3 \mathrm{ml}$ hexamethyldisilazane and $0.1 \mathrm{ml}$ trimethylchlorosilane were added. The suspension was mixed vigorously for $1 \mathrm{~min}$ (Vari-Whirl, Van Waters and Rogers, Inc., San Francisco, Calif.) and allowed to stand at room temperature for $15 \mathrm{~min}$ for conversion to trimethylsilyl (TMS) ether derivatives. The mixture was centrifuged at $3,000 \mathrm{rpm}$ for $15 \mathrm{~min}$ to remove the $\mathrm{NH}_{4} \mathrm{Cl}$ precipitate, and the supernate was taken to dryness under a nitrogen stream at room temperature. The dry derivatives were taken up in $0.3 \mathrm{ml}$ ethyl ether, and $3 \mu 1$ of the solution injected onto the column. All reagents and standards were chromatographic grade and purchased either from Sigma Chemical Co., St. Louis, Mo. or Applied Science Laboratories Inc., State College, $\mathrm{Pa}$.

A model F \& M 402 dual-column gas chromatograph and a Hewlett-Packard model $5700 \mathrm{~A}$ gas chromatograph, both with flame ionization detectors and computerized peak integrators (Hewlett-Packard Co., Avondale Div., Avondale, Pa.), were used. All analyses were performed on columns packed with $3 \%$ SE-30 on acid-washed, silanized, 60/80-mesh Chromosorb W (Applied Science Laboratories

TABLE I

Serum Samples Analyzed by GLC*

\begin{tabular}{lcl}
\hline \multicolumn{1}{c}{ Organism isolated } & $\begin{array}{c}\text { Number } \\
\text { analyzed }\end{array}$ & \multicolumn{1}{c}{ Results } \\
\hline Candida albicans & 7 & Positive (7) \\
Escherichia coli & 4 & Negative \\
Aerobacter aerogenes & 3 & Negative \\
Klebsiella pneumoniae & 2 & Negative \\
Pseudomonas aeruginosa & 6 & Negative \\
Shigella shiga & 1 & Negative \\
Diplococcus pneumoniae & 3 & Negative \\
Mycobacterium leprae & 5 & Negative \\
\hline
\end{tabular}

* All serum samples, with the exception of those from patients with leprosy, were obtained at the time of proven bacteremia.
Inc.). The carrier gas was nitrogen at a flow rate of 40 $\mathrm{ml} / \mathrm{min}$. The injector and detector were maintained at $210^{\circ} \mathrm{C}$; the column oven temperature was $170^{\circ} \mathrm{C}$. Mass spectroscopy was performed by Dr. John Wright (Departments of Biology and Medicine, University of California at San Diego) who used an LKB mass spectrophotometer-gas chromatograph unit (LKB Instruments, Inc., Rockville, Md.). Retention times of peaks measured in seconds were expressed relative to the retention time of palmitic acid which was assigned a value of one. Areas of abnormal peaks were recorded as a percentage of the area of the palmitic acid peak for each chromatogram. Mean areas of abnormal peaks, relative to palmitic acid, were determined for each group of patients and compared by the Student's $t$ test.

\section{RESULTS}

Three large peaks labeled A, B, and C in Fig. 1 were found in all sera. Two samples were dialyzed overnight against running tap water before hydrolysis, with no effect on the resulting chromatograms. Two other samples were then hydrolyzed and neutralized, as previously described, but extracted with three equal volumes of chloroform. The chloroform layer and remaining aqueous fraction were dried and silylated separately. Chromatogaphy of derivatives of both fractions demonstrated that peaks $A, B$, and $C$ were extracted into the chloroform phase.

A mixture of fatty acid methyl esters (Sigma Chemical Co.) was chromatographed showing three peaks with the same retention times as peaks $\mathrm{A}, \mathrm{B}$, and $\mathrm{C}$ of normal serum. Cochromatography and mass spectroscopy confirmed that peak A represented palmitic acid methyl ester, B constituted a mixture of oleic and linoleic acid methyl esters, and $\mathrm{C}$ was the methyl ester of stearic acid. Some normal sera also contained peaks that cochromatographed with other short- and medium-chain fatty acids, but these peaks were small and inconsistent.

Six different organisms (Table I) were represented among the 19 sera corresponding to bacterial septicemias. The chromatograms of the 19 sera from patients with nonfungal sepsis and the five patients with leprosy were virtually identical to chromatograms of normal serum (Fig. 1). The peak integrator was not used for the chromatograms of the first seven control sera and the bacteremia and leprosy chromatograms, for these were indistinguishable and demonstrated only peaks A, B, and C (Fig. 1). Seven chromatograms of sera from patients with $C$. albicans sepsis were found to be significantly different from normal and identical to each other (Fig. 2). The peak integrator and timer was used to measure the retention time and areas of the abnormal peaks $D, E, F$, and $G$, relative to the normal peaks in nine normal control sera. The abnormal peaks were found to have retention times relative to palmitic acid of approximately 0.46 (D), 0.56 (E), 0.70 (F), and 0.81 (G). All seven chromatographically positive sera 


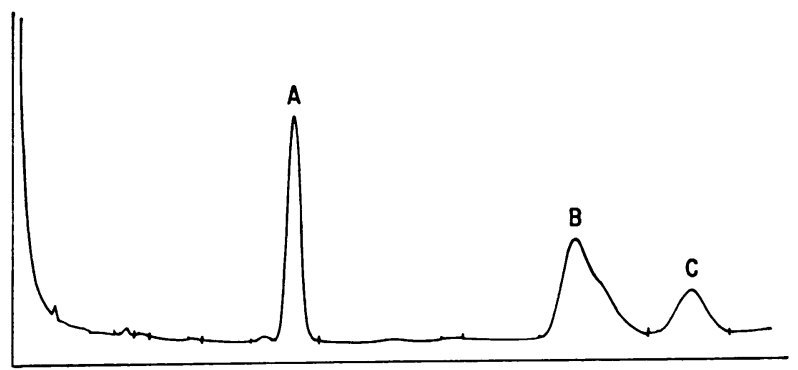

FIGURE 1 Normal human serum. Gas chromatogram of normal serum on 6-foot glass column packed with $3 \%$ SE-30 on Chromosorb W. Carrier gas was nitrogen at $40 \mathrm{ml} / \mathrm{min}$. Injector and detector temperatures were $210^{\circ} \mathrm{C}$; column oven temperature was $170^{\circ} \mathrm{C} .2 \mu 1$ of methyl ester TMS ether derivatives injected onto column. Peak A represents palmitic acid methyl ester, peak B a mixture of oleic and linoleic acid methyl esters, and peak $C$ the methyl ester of stearic acid.

also contained the three normal peaks (methyl esters of palmitic, oleic, linoleic, and stearic acids) but with greater variability in peak areas.

Candidemia in the six patients resulted from indwelling catheters in four cases, a burn in one case, and renal papillary necrosis in the other. Only one was a diabetic and none received hyperalimentation. The normal chromatograms from 19 bacteremia patients provided negative controls for these patients' underlying diseases and associated illnesses, as well as for bacteremia. A list of these conditions ${ }^{2}$ emphasizes the variety of illnesses that does not alter the normal chromatogram.

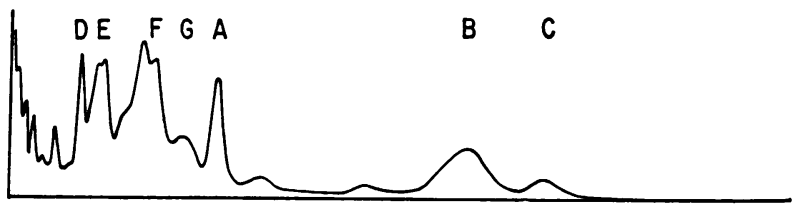

FIGURE 2 Serum from a patient with $C$. albicans sepsis. Chromatogram of methyl ester TMS ether derivative of serum from patient J5 with blood culture positive for $C$. albicans. Column and temperature same as Fig. 1. $3 \mu \mathrm{l}$ injected onto column. Note additional peaks D, E, F, and $G$ that were not present in normal serum. Relative retention times of additional peaks (palmitic acid, 1.0) were $0.46(\mathrm{D}), 0.56(\mathrm{E}), 0.69(\mathrm{~F})$, and $0.81(\mathrm{G})$.

${ }^{2}$ Pancreatitis, diabetes mellitus, carcinoma (lung and prostate), malignant lymphoma stage IVB, myelocytic leukemia, infarcts (lung, spleen, and brain), disseminated herpes, acute tubular necrosis secondary to incompatible blood transfusion, uremic pericarditis, sickle cell disease, cholelithiasis, osteomyelitis, pyelonephritis, thrombophlebitis, perforated abdominal viscera (stomach, duodenum, ileum), gangrenous ileum secondary to strangulated hernia, alcoholism, Laënnec's cirrhosis, hepatocellular disease, renal vein thrombosis, myocardial infarct, congestive heart failure, bilirubinemia, "flail" chest, bleeding esophageal varices, porta-caval shunt, traumatic rupture of the spleen with intra-abdominal hemorrhage, and subdural hematoma.

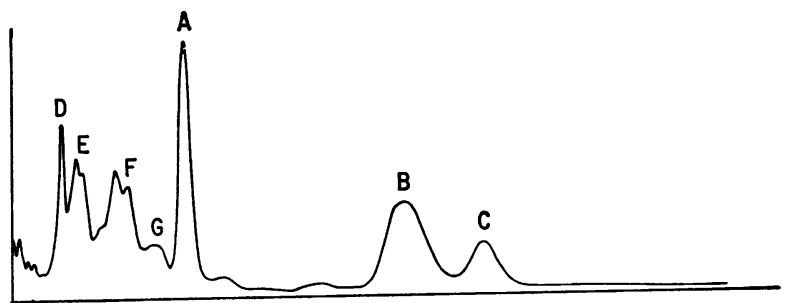

FIGURE 3 Serum used as culture medium for $C$. albicans. $C$. albicans was inoculated into normal serum and incubated for $72 \mathrm{~h}$. The culture was centrifuged and filtered and methyl ester TMS ether derivatives were prepared from the cell-free serum; $3 \mu l$ of the derivative were injected onto the column. Column and temperatures same as Fig. 1. Note that this chromatogram is identical to that of the patient with candidemia shown in Fig. 2. Relative retention times of additional peaks (palmitic acid, 1.0) were 0.46 (D), $0.56(\mathrm{E}), 0.69(\mathrm{~F})$, and $0.81(\mathrm{G})$.

To determine further whether these differences were secondary to $C$. albicans septicemia rather than to other factors peculiar to these paitents, we chromatographed serum cultures of $C$. albicans. As shown in Fig. 3, the chromatogram of the cell-free serum in which the yeast had grown was identical to the chromatograms of serum from the six patients with $C$. albicans septicemia. The chromatogram of the uninoculated serum control was unchanged from the normal.

The next experiment indicated that these peaks were components of the yeast cell. Chromatograms of $C$. albicans harvested from overnight cultures in YNB, Fig. 4, contained all the abnormal peaks found in the serum from

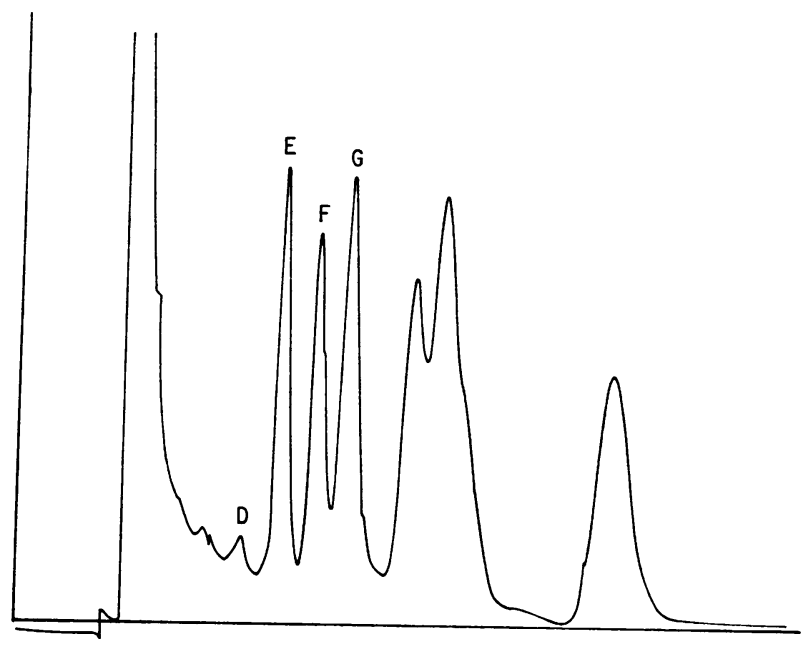

Figure 4 Washed $C$. albicans. $C$. albicans was inoculated into $50 \mathrm{ml}$ of YNB and incubated for $24 \mathrm{~h}$. The cells were collected by centrifugation at $10,000 \mathrm{~g}$, washed three times with $10 \mathrm{vol}$ of $0.15 \mathrm{M} \mathrm{NaCl}$, dried overnight in vacuo at $40^{\circ} \mathrm{C}$, suspended in $1 \mathrm{ml}$ distilled water, and chromatographed by the same technique as the serum samples. Peaks $D, E, F$, and $G$ cochromatograph exactly with peaks $D$, $E, F$, and $G$ in candidemic serum. 
TABLE II

Peak Areas Relative to Palmitic Acid (Percent \pm 2 SEM)

\begin{tabular}{|c|c|c|c|c|c|}
\hline Serum & $\begin{array}{c}\text { No. of } \\
\text { samples }\end{array}$ & $\begin{array}{c}\mathrm{D} \\
0.45-0.49\end{array}$ & $\underset{0.54-0.57}{E}$ & $\begin{array}{c}F \\
0.68-0.72\end{array}$ & $\underset{0.80-0.81}{G}$ \\
\hline Candidemia & 7 & $72 \pm 45$ & $76 \pm 55$ & $109 \pm 50$ & $42 \pm 28$ \\
\hline Thrush & 11 & $\begin{array}{c}4 \pm 2 \\
(P<0.01)^{*}\end{array}$ & $5.4 \pm 3.3$ & $\begin{array}{c}9.1 \pm 3 \\
(P<0.001)\end{array}$ & $\begin{array}{c}1.2 \pm 7.6 \\
(P<0.05)\end{array}$ \\
\hline Normal & 9 & $\begin{array}{c}2.9 \pm 3.0 \\
(P<0.01)\end{array}$ & $\begin{array}{l}1.5 \pm 1.8 \\
(P<0.01)\end{array}$ & $\begin{array}{c}3.5 \pm 3.1 \\
(P<0.001)\end{array}$ & 0 \\
\hline Deep invasive & 5 & $23 \pm 11$ & $41 \pm 19$ & $64 \pm 41$ & $37 \pm 37$ \\
\hline
\end{tabular}

* When compared to candidemia.

candidemia patients. 1-ml samples of the culture supernate from which the cells were harvested and from the supernatant wash fluid contained only very small amounts of the abnormal peaks. The peaks found in chromatograms of the whole cells, labeled D, E, F, and G in Fig. 4 , cochromatographed perfectly with peaks $D, E, F$, and $\mathrm{G}$ in the serum from one of the patients with candidemia. Serum from one of the patients with candidemia was hydrolyzed and neutralized, as previously described, but was then extracted with three equal volumes of chloroform. The chloroform and remaining aqueous fractions were dried and silylated separately. As demonstrated

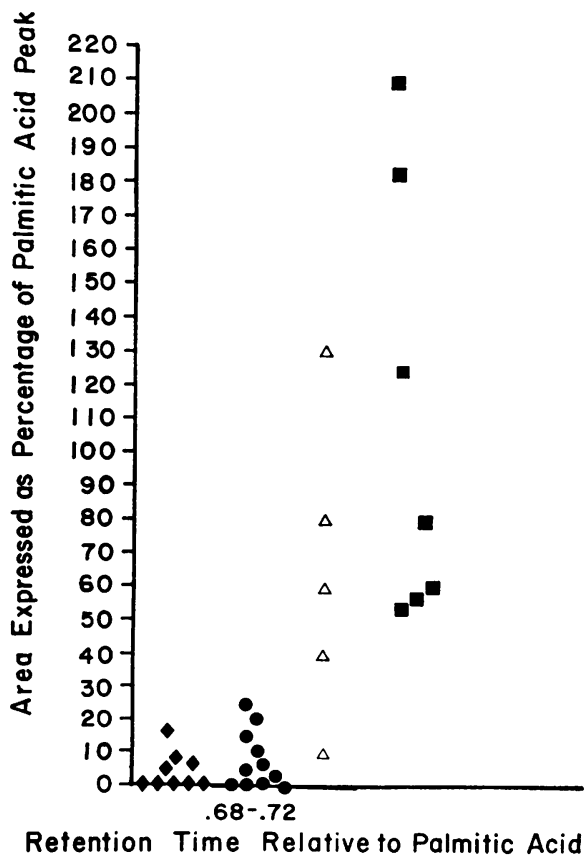

FIgURE 5 Distribution of areas relative to palmitic acid of peak $F(0.70)$. Relative peak size was calculated and plotted from chromatograms of serum from 6 patients with candidemia ( 7 samples), 4 patients with deep invasive candidiasis $\Delta$ (5 samples), 11 patients with thrush $\bullet$, and 9 normal controls $\diamond$. previously for normal serum, peaks $\mathrm{A}, \mathrm{B}$, and $\mathrm{C}$ were extracted into the chloroform phase. The abnormal peaks $D, E, F$, and $G$ remained in the aqueous phase suggesting that they might not be lipids. Because these peaks represented substances resistant to concentrated acid hydrolysis, it seemed unlikely that they were proteins. Accordingly, various sugars were subjected to silylation and chromatography. A TMS derivative of mannose (Calbiochem, Los Angeles, Calif.) had two peaks on GLC, probably representing isomers, which were found to cochromatograph with peaks $F(0.70)$ and $G(0.81)$ that were present on chromatograms of both candidemic serum and washed cells of $C$. albicans. These two abnormal peaks seem to represent isomers of mannose; the remaining abnormal peaks remain to be identified.

Chromatograms from patients with superficial candidiasis were similar to normals. Serum from two of the four patients with deep-invasive candidiasis and negative blood cultures were identical chromatographically to the fungemia patients. Chromatograms of serum obtained from both of these patients became normal after eradication of infection. One was normal 1 week after removal of an infected intrascrotal prosthesis, and the other was normal a few weeks after debridement and healing of an infected sternal wound. The other two patients with deep-invasive candidiasis had chromatographic patterns identical with those of superficial candidiasis. One patient with candidemia was studied again 5 days after blood cultures became negative. The chromatogram was almost normal. The small abnormal peaks that remained were indistinguishable from the thrush group.

We were unable to classify two paients with candidiasis and sterile blood cultures. One with widely metastatic carcinoma of the lung had severe oral mucocutaneous thrush, and cultures of bronchial brushings produced heavy growth of $C$. albicans. The other had multiple traumatic wounds, more than 100,000 colonies of $C$. albicans per $\mathrm{ml}$ of urine, and several bronchial washings with a significant growth of $C$. albicans. Chromatograms 
of serum from these two patients were significantly different from those with candidemia but abnormal when compared to those of patients with thrush. They probably belong in the deep-invasive group, but were eliminated from calculations because we had no unequivocal evidence of parenchymal disease.

Because the chromatograms of several patients with superficial candidiasis and of an occasional normal control had small early peaks with relative retention times to palmitic acid similar to the four "abnormal" peaks found during fungemia, the four groups (normals, superficial candidiasis, deep-seated candidiasis, and candidemia) were compared by taking the areas of peaks with retention times of approximately 0.46 (D), 0.56 (E), $0.70(F)$, and 0.81 (G) in each chromatogram and expressing them as a percentage of the palmitic peak area. As shown in Table II, there was good separation of mean values ( $\pm 2 \mathrm{SEM}$ ) for each peak in the group with candidemia from the normal controls and from the patients with superficial infections. The mean areas of peaks $D, E, F$, and $G$ from candidemic patients were significantly different from those of normals, and the areas of peaks D, F, and G from the group with candidemia were statistically different from the group with thrush. The two patients with deep-invasive candidiasis whose abnormal chromatograms fit with the fungemia group, are responsible for the overlap between fungemia and deep-invasive disease. The area of peak $F$, the 0.70 peak, of each sample expressed as the percentage of palmitic acid, is shown in Fig. 5. The area of peak F identified all patients with significant Candida infection because it was greater than $50 \%$ of the palmitic acid peak only in patients with fungemia or deep-invasive candidiasis.

\section{DISCUSSION}

We discovered in these experiments that gas chromatograms of candidemic sera were so characteristic that GLC can be used to diagnose candidemia long before blood cultures become positive. Underlying noninfectious diseases cannot have been responsible for these GLC patterns in candidemia because chromatograms of serum from patients with the same diseases in the absence of Candida septicemia were normal. The abnormal peaks were not caused by antibiotics, hyperalimentation, or abnormal glucose metabolism because no patient received antibiotics or hyperalimentation and only one was a diabetic. The significance of the results was reinforced by our finding that the same compounds appeared in chromatograms of sera inoculated with growing cultures of $C$. albicans. Since bacterial septicemias have thus far never produced abnormal chromatograms, it is clear that our technique identified products unique to Candida septicemia. That these products were part of the
Candida cell was illustrated by production of the same chromatographic pattern on chromatography of washed cells of $C$. albicans grown in the absence of serum. Although not identified, cochromatography suggests that these abnormal peaks might be derivatives of mannose.

We also tried to learn if the preseptic state, i.e., local deep tissue invasion before the dissemination of Candida, could be recognized and distinguished from superficial mucosal colonization. Examination of 11 patients with thrush and 4 patients with significant deep-tissue invasion showed that candidemia and the preseptic state can be distinguished from superficial colonization. Chromatograms of sera from two patients with deep-invasive candidiasis were indistinguishable from those with candidemia and became normal after the infections were cured. The patient with candidemia, who was available for follow-up study 5 days after the septicemia had cleared, also reverted to virtually normal. Superficial colonization did, however, produce small peaks with relative retention times similar to the peaks in fungemic sera, but the ratio of peak $F(0.70)$ to palmitic acid distinguished candidemia and deep-invasive candidiasis from simple thrush and normal controls (Table II and Fig. 5).

Mitruka, Jonas, and Alexander (4) reported that the specific organism causing bacteremia in rats and mice could be recognized by GLC of serum, but they examined serum and tissue with extraordinarily heavy infections rarely encountered in patients $\left(10^{4}-10^{7}\right.$ bacteria per $\mathrm{ml}$ of blood and $10^{4}-10^{\circ}$ bacteria per $g$ of tissue). This would account for our negative results in patients with bacteremia.

The difficulties in diagnosing disseminated candidiasis by clinical and cultural methods (6) have led to the search for more rapid and accurate diagnostic methods $(7,8)$. GLC possesses the advantages of speed and simplicity. Findings reported here should encourage others to use GLC so that its general applicability in the rapid diagnosis of Candida septicemia may be evaluated in large numbers of patients. Further work is in progress in this laboratory to develop gentler methods of preparation and to increase sensitivity in the adaptation of GLC for use in rapid diagnosis of bacteremia.

Aside from their potential diagnostic significance, these findings may give important insight into the pathogenesis of Candida infections. They indicate that in disseminated candidiasis, like cryptococcosis (9), pneumococcal pneumonia (10), and gram-negative bacterial infections (11), microbial cell products are regularly present in the circulation. In the cryptococcal and bacterial infections, these products are sugars or lipopolysaccharides that withstand degradation in the body fluids and contribute to virulence and toxicity of the organisms. On the basis of the present study, it is reasonable to 
consider that similar products, possibly Candida sugars, might contribute to the pathogenicity of disseminated candidiasis.

\section{REFERENCES}

1. Davis, C. E., S. D. Freedman, H. Douglas, and A. I. Braude. 1969. Analysis of sugars in bacterial endotoxins by gas-liquid chromatography. Anal. Biochem. 28: 243-256.

2. Yamakawa, T., and N. Ueta. 1964. Gas-chromatographic studies of microbial components. I. Carbohydrate and fatty acid constitution of Neisseria. Jap. J. Expt. Med. 34: 361-374

3. Doelle, H. W., and G. J. Manderson. 1969. Preparation of extracts of culture liquids for gas-chromatographic determination of acidic fermentation products. Antonie Van Leeuwenhoek J. Microbiol. Serol. 35: 467-478.

4. Mitruka, B. M., A. M. Jonas, and M. Alexander. 1970. Rapid detection of bacteremia in mice by gas chromatography. Infect. Immun. 2: 474-478.

5. Mitruka, B. M. 1970. Biochemical aspects of Diplococ- cus pneumoniae infections in laboratory rats. Yale $J$. Biol. Med. 44 : 253-264.

6. Hart, P. D., E. Russell, Jr., and J. S. Remington. 1969. The compromised host and infection. II. Deep fungal infection. J. Infect. Dis. 120: 169-191.

7. Rosner, F., F. D. Gabriel, C. L. Taschdjian, M. D. Cuesta, and P. J. Kozinn. 1971. Serologic diagnosis of systemic candidiasis in patients with acute leukemia. Am. J. Med. 51: 54-61.

8. Remington, J. S., J. D. Gaines, and M. A. Gilmer. 1972. Demonstration of candida precipitins in human sera by counterimmunoelectrophoresis. Lancet. 1: 413.

9. Bennett, J. E., and H. F. Hasenclever. 1965. Cryptococcus neoformans polysaccharide: studies of serologic properties and role in infection. J. Immunol. 94: 916920.

10. Park, W. H., and G. Cooper. 1928. Antipneumococcic serum in lobar pneumonia: method of administration and dosage. JAMA. 90: 1349-1351.

11. Braude, A. I., J. L. Jones, and H. Douglas. 1963. The behaviour of Escherichia coli endotoxin (Somatic antigen) during infectious arthritis. J. Immunol. 90: 297311. 